\title{
Mild solution for a stochastic partial differential equation with noise
}

\author{
HABIB DJOURDEM \\ Laboratory of Fundamental and Applied \\ Mathematics of Oran (LMFAO) \\ University of Oran1, Ahmed Benbella \\ El M'Naouer -31000 Oran \\ ALGERIA \\ djourdem.habib7@gmail.com
}

\author{
NOUREDDINE BOUTERAA \\ Laboratory of Fundamental and Applied \\ Mathematics of Oran (LMFAO) \\ University of Oran1, Ahmed Benbella \\ El M'Naouer -31000 Oran \\ ALGERIA \\ bouteraa-27@hotmail.fr
}

\begin{abstract}
This paper focuses on the study of the existence of a mild solution to time and space-fractional stochastic equation perturbed by multiplicative with noise. We use stochastic analysis techniques, fractional calculus, semigroup theory and we apply Sadovskii's fixed point theorem to prove our main result.
\end{abstract}

Key-Words: stochastic equation, mild solution, fractional derivative, Hausdorff measure of non-compacteness, Sadovskii's fixed point

Received: April 22, 2020. Revised: August 1, 2020, Accepted: September 9, 2020. Published: September 16, 2020.

\section{Introduction}

In this paper, we are interested in the existence of solutions for nonlinear fractional difference equations

$$
\begin{gathered}
{ }^{c} D_{t}^{\alpha}[u-h(u)]=\Delta u(t)+u \cdot \nabla u+g(u) W(t), \\
x \in D, t>0,
\end{gathered}
$$

subject to the initial condition

$$
u(x, 0)=u_{0}(x), x \in D, t=0,
$$

and the Dirichlet boundary conditions

$$
u(x, t)=0, x \in \partial D,
$$

where $D \subset \mathbb{R}^{d}, u(x, t)$ represents the velocity field of the fluid, the state $u(\cdot)$ takes values in a separable real Hilbert space $H$ with inner product $\langle\cdot, \cdot\rangle$, the term $g(u) W(t)=g(u) \frac{d}{d t} W(t)$ describes a state dependent random noise, where $W(t)_{t \in[0, T]}$ is a $F_{t}$-adapted Wiener process defined in completed probability space $(\Omega, F, P)$ with expectation $E$ and associate with the normal filtration $F_{t}=$ $\sigma\{W(s): 0 \leq s \leq t\}$. The operator $\Delta$ is the Laplacian. Here, ${ }^{c} D_{t}^{\alpha}$ denotes the Caputo type derivative of order $\alpha(0<\alpha<1)$ for the function $u(x, t)$ with respect time $t$ which is defined by

$$
\left\{\begin{array}{cc}
{ }^{c} D_{t}^{\alpha} u(t, x)=\frac{1}{\Gamma(1-\alpha)} \int_{0}^{t} \frac{\partial u(x, s)}{\partial s} \frac{d s}{(t-s)^{\alpha}}, 0<\alpha<1, \\
\frac{\partial u(t, x)}{\partial t}, & \alpha=1,
\end{array}\right.
$$

where $\Gamma(\cdot)$ stands for the Gamma function $\Gamma(\alpha)=\int_{0}^{\infty} t^{\alpha-1} e^{-t} d t$.
There has been a widespread interest during the last decade in constructing a stochastic integration theory with respect to fractional Brownian motion (FBM) and solving stochastic differential equations driven by FBM. In fact, stochastic perturbation factors, such as precipitation, absolute humidity, and temperature, have a significant impact on the infection force of all types of virus diseases to humans. Taking this into consideration enables us to present randomness into deterministic biological models to expose the environmental variability effect, whether it is environmental fluctuations in parameters or random noise in the differential systems [18]. Stochastic population dynamics perturbed by white noise (or Brownian motion) has been studied extensively by many authors $[1,12,13]$. It has been investigated in [14] that an environmental Brownian noise can suppress explosions in population dynamics. Yuan et al. [28] discussed the results of stochastic viral infection, immune response dynamics and analyzed the human immunodeficiency virus infection.

On the other hand, time-fractional differential equations are found to be quite effective in modelling anomalous diffusion processes as its can characterize the long memory processes $[6,19,27,22]$. Hence, Burgers equation with time-fractional can be adapted to describe the memory effect of the wall friction through the boundary layer [8]. Furthermore, the analytical solutions of the time- and space-fractional Burgers equations have been investigated by variational iteration method and Adomian decomposition method [15]. Some relevant studies can be found in $[4,21,25,26,29]$. 
The existence of solution for partial neutral integro-differential equation with infinite delay in infinite dimensional spaces has been extensively studied by many authors, see for example [16]. Ezzinbi and al. [7] investigated the existence and regularity of solutions for some partial functional integro-differential equations in Banach spaces. Cui and Yan [5] investigated the existence of mild solutions for a class of fractional neutral stochastic integro-differential equations with infinite delay in Hilbert spaces by means of Sadovskiis fixed point theorem. In particular, the stability theory of stochastic differential equations has been popularly applied in variety fields of science and technology. Several authors have established the stability results of mild solutions for these equations by using various techniques, we refer the reader to $[3,10,11,17]$.

The main contribution of this paper is to establish the existence of mild solution for the problem (1)(3). Using mainly the Sadovskii's fixed point theorem. The rest of the paper organised as follows, In Section 2 , we will introduce some notations and preliminaries, which play a crucial role in our theorem analysis. In Section 3, the existence results on a mild solutions are derived.

\section{Preliminaries}

In this section, we give some notions and certain important preliminaries, which will be used in the subsequent discussions. Let $\left(\Omega, F,\{F\}_{t \geq 0}, P\right)$ be a filtered probability space with a normal filtration, where $P$ is a probability measure on $(\Omega, F)$ and $F$ is the Borel $\sigma$-algebra. Let $\{F\}_{t>0}$ satisfying that $F_{0}$ contains all $P$-null sets. The operator $A$ is the infinitesimal generator of a strongly continuous semigroup on a separable real Hilbert space $H$.

Denote the basic functional space $L^{p}(D), 1 \leq p<$ $\infty$ and $H^{s}(D)$ by the usual Lebesgue and Sobolev space, respectively. We assume that $A$ is the negative Laplacian $-\Delta$ in a bounded domain with zero Dirichlet boundary conditions in Hilbert space $H=L^{2}(D)$, which are given by

$$
A=-\triangle, D(A)=H_{0}^{1}(D) \cap H^{2}(D),
$$

since the operator $A$ is self-adjoint, i.e., there exist the eigenvectors $e_{k}$ corresponding to eigenvalues $\lambda_{k}$ such that

$$
A e_{k}=\lambda_{k} e_{k}, e_{k}=\sqrt{2} \sin (k \pi), \lambda_{k}=\pi^{2} k^{2}, k \in \mathbb{N}^{+} .
$$

For any $\sigma>0$, let $H^{\sigma}$ be the domain of the frac- tional power $A^{\frac{\sigma}{2}}=(-\Delta)^{\frac{\sigma}{2}}$, which can be defined by

$$
\sigma>0, \quad A^{\frac{\sigma}{2}} e_{k}=\gamma_{k}^{\frac{\sigma}{2}} e_{k}, k=1,2, \ldots
$$

and

$$
\begin{aligned}
H^{\sigma}=D\left(A^{\frac{\sigma}{2}}\right)=\left\{v \in L^{2}(D),\right. \\
\left.\|v\|_{H^{\sigma}}^{2}=\sum_{k=1}^{\infty} \gamma_{k}^{\frac{\sigma}{2}} v_{k}^{2}<\infty\right\}
\end{aligned}
$$

where $v_{k}=\left\langle v, e_{k}\right\rangle$ with the inner product $\langle\cdot, \cdot\rangle$ in $L^{2}(D)$, the norm $\left\|H^{\sigma} v\right\|=\left\|A^{\frac{\sigma}{2}} v\right\|$, the bilinear operator $B(u, v)=u \cdot \nabla v$ and $\mathcal{D}(B)=H_{0}^{1}(D)$ with the slight abuse of notation $B(u)=B(u, u)$. Then we can rewrite the equations (1)-(3) as follows in the abstract form

$$
\left\{\begin{aligned}
{ }^{c} D_{t}^{\alpha}[u(t)-h(u(t))]= & A u(t)+B(u(t)) \\
& +g(u(t)) \frac{d W(t)}{d t}, t>0, \\
u(0)=u_{0}, &
\end{aligned}\right.
$$

where $\{W(t), t \geq 0\}$ is a $Q$-Wiener process with linear bounded covarience operator $Q$ such that a trace class operator $Q$ denote $\operatorname{Tr}(Q)=\sum_{k=1}^{\infty} \lambda_{k}<\infty$, which satisfies that $Q e_{k}=\lambda_{k} e_{k}, k=1,2, \ldots$, then the Wiener process is given by

$$
W(t)=\sum_{k=1}^{\infty} \sqrt{\lambda_{k}} \beta_{k}(t) e_{k},
$$

where $\left\{\beta_{k}\right\}_{k=1}^{\infty}$ is a sequence of real-valued standard Brownian motions.

Let $L_{0}^{2}=L^{2}\left(Q^{\frac{1}{2}}(H), H\right)$ be a Hilbert-Schmidt space of operators from $Q^{\frac{1}{2}}(H)$ to $H$ with the norm

$$
\|\phi\|_{L_{0}^{2}}=\left\|\phi Q^{\frac{1}{2}}\right\|_{H^{\sigma}}=\left(\sum_{n=1}^{\infty} \phi Q^{\frac{1}{2}} e_{n}\right)^{\frac{1}{2}}
$$

i.e.,

$$
L_{0}^{2}=\left\{\phi \in L(H): \sum_{n=1}^{\infty}\left\|\lambda_{n}^{\frac{1}{2}} \phi Q^{\frac{1}{2}} e_{n}\right\|^{2}<\infty\right\},
$$

where $L(H)$ is the space of bounded linear operators from $H$ to $H$.

For an arbitrary Banach space $B$, we denote

$$
\begin{gathered}
\|v\|_{L^{p}(\Omega, B)}=\left(E\|v\|_{B}^{p}\right)^{\frac{1}{p}}, \forall v \in L^{p}(\Omega, F, P, B), \\
\text { for any } p \geq 2 .
\end{gathered}
$$

We shall also need the following result with respect to the operator $A_{\nu}$ (see [22]). 
Lemma 1. For any $\nu>0$, an analytic semigroup $T(t)=e^{-t A_{\nu}}, t \geq 0$ is generated by the operator $A_{\nu}$ on $L^{p}$, there exists a constant $C_{\nu}$ dependent on $\nu$ such that

$$
\left\|A_{\nu} T(t)\right\|_{L\left(L^{p}\right)} \leq C_{\nu} t^{-\nu}, t>0,
$$

in which $L(B)$ denotes the Banach space of all bounded operators from $K$ to itself.

Next we will introduce the following lemma to estimate the stochastic integrals, which contains the Burkhoder-Davis-Gundy's inequality.

Lemma 2. [9] For any $0 \leq t_{1}<t_{2} \leq T$ and $p \geq 2$ and for any predictable stochastic process $v:[0, T] \times \Omega \rightarrow L_{0}^{2}$ which satisfies

$$
E\left[\left(\int_{0}^{T}\|v(s)\|_{L_{0}^{2}}^{2} d s\right)^{\frac{p}{2}}\right]<\infty
$$

then, we have

$E\left[\left\|\int_{t_{1}}^{t_{2}} v(s) d W(s)\right\|^{p}\right]<C(p) E\left[\left(\int_{t_{1}}^{t_{2}}\|v(s)\|_{L_{0}^{2}}^{2} d s\right)^{\frac{p}{2}}\right]$,

where $C(p)>0$ is a constant depending only on $p$.

Inspired by the definition of the mild solution to the time-fractional differential equations (see [19]), we give the following definition of mild solution for our time-fractional stochastic equation.

Definition 3. An $F_{t}$-adapted stochastic process $(u(t), t \in[0, T])$ is called a mild solution to (5) if the following integral equation is satisfied

$$
\begin{aligned}
u(t)= & E_{\alpha}(t) u_{0}+h(u(t)) \\
& +\int_{0}^{t}(t-s)^{\alpha-1} E_{\alpha, \alpha}(t-s) B(u(s)) d s \\
& +\int_{0}^{t}(t-s)^{\alpha-1} E_{\alpha, \alpha}(t-s) g(u(s)) d W(s),
\end{aligned}
$$

where the generalized Mittag-Leffler operators $E_{\alpha}(t)$ and $E_{\alpha, \alpha}(t)$ are defined, respectively, by

$$
E_{\alpha}(t)=\int_{0}^{\infty} \zeta_{\alpha}(\theta) T\left(t^{\alpha} \theta\right) d \theta
$$

and

$$
E_{\alpha, \alpha}(t)=\int_{0}^{\infty} \alpha \theta \zeta_{\alpha}(\theta) T\left(t^{\alpha} \theta\right) d \theta
$$

where $T(t)=e^{-t A}, t \geq 0$ is an analytic semi group generated by the operator $-A$ and the Mainardi's Wright-type function with $\alpha \in(0,1)$ is given by

$$
\zeta_{\alpha}(\theta)=\sum_{k=0}^{\infty} \frac{(-1)^{k} \theta^{k}}{k ! \Gamma(1-\alpha(1+k))} .
$$

Lemma 4. [5] For any $\alpha \in(0,1)$ and $-1<\nu<\infty$, it is not difficult to verity that

$$
\zeta_{\alpha}(\theta) \geq 0 \text { and } \int_{0}^{\infty} \theta^{\nu} \zeta_{\alpha}(\theta) d \theta=\frac{\Gamma(1+\nu)}{\Gamma(1+\alpha \nu)},
$$

for all $\theta \geq 0$.

The operators in (7) and $\left\{E_{\alpha}(t)\right\}_{t \geq 0}$ and $\left\{E_{\alpha, \alpha}(t)\right\}_{t \geq 0}$ have the following properties.

Lemma 5. For any $E_{\alpha}(t)$ and $E_{\alpha, \alpha}(t)$ are linear and bounded operators. Moreover, for $0<\alpha<1$ and $0 \leq \nu<2$, there exists a constant $C>0$ such that $E_{\alpha}(t)$ and $E_{\alpha, \alpha}(t)$ are defined, respectively, by

$$
\left\|E_{\alpha}(t) \chi\right\|_{H^{\nu}} \leq C t^{-\frac{\alpha \nu}{2}}\|\chi\|, \quad\left\|E_{\alpha, \alpha}(t) \chi\right\|_{H^{\nu}} \leq C t^{-\frac{\alpha \nu}{2}}\|\chi\| .
$$

Proof. For $T>0$ and $0 \leq \nu<2$, by means of Lemma 1 and Lemma 4, we have

$$
\begin{aligned}
\left\|E_{\alpha}(t) \chi\right\|_{H^{\nu}} & \leq \int_{0}^{\infty} \zeta_{\alpha}(\theta)\left\|A_{\nu} T\left(t^{\alpha} \theta\right) \chi\right\| d \theta \\
& \leq \int_{0}^{\infty} C_{\nu} t^{-\frac{\alpha \nu}{2}} \theta^{-\nu} \zeta_{\alpha}(\theta)\|\chi\| d \theta \\
& =\frac{C_{\nu} \Gamma(1-\nu)}{\Gamma(1-\alpha \nu)} t^{-\frac{\alpha \nu}{2}}\|\chi\|, \quad \chi \in L^{2}(D),
\end{aligned}
$$

and

$$
\begin{aligned}
\| E_{\alpha, \alpha}(t) & \chi\left\|_{H^{\nu}} \leq \int_{0}^{\infty} \alpha \theta \zeta_{\alpha}(\theta)\right\| A_{\nu} T\left(t^{\alpha} \theta\right) \chi \| d \theta \\
& \leq \int_{0}^{\infty} C_{\nu} \alpha t^{-\frac{\alpha \nu}{2}} \theta^{1-\nu} \zeta_{\alpha}(\theta)\|\chi\| d \theta \\
& =\frac{C_{\nu} \alpha \Gamma(2-\nu)}{\Gamma(1-\alpha \nu)} t^{-\frac{\alpha \nu}{2}}\|\chi\|, \quad \chi \in L^{2}(D),
\end{aligned}
$$

so, $E_{\alpha}(t)$ and $E_{\alpha, \alpha}(t)$ are linear and bounded operators. The proof is completed. 
Lemma 6. For any $t>0$, the operators $E_{\alpha}(t)$ and $E_{\alpha, \alpha}(t)$ are strongly continuous. Moreover, for $0<$ $\alpha<1$ and $0 \leq \nu<2$ and $0 \leq t_{1}<t_{2} \leq T$, there exists a constant $C>0$ such that

$$
\left\|\left(E_{\alpha}\left(t_{2}\right)-E_{\alpha}\left(t_{1}\right)\right) \chi\right\|_{H^{\nu}} \leq C\left(t_{2}-t_{1}\right)^{\frac{\alpha \nu}{2}}\|\chi\|,
$$

and

$\left\|\left(E_{\alpha, \alpha}\left(t_{2}\right)-E_{\alpha, \alpha}\left(t_{1}\right)\right) \chi\right\|_{H^{\nu}} \leq C\left(t_{2}-t_{1}\right)^{\frac{\alpha \nu}{2}}\|\chi\|$.

Proof. For any $0<T_{0} \leq t_{1}<t_{2} \leq T$, it is easy to deduce that

$$
\int_{t_{1}}^{t_{2}} \frac{d T\left(t^{\alpha} \theta\right)}{d t} d t=T\left(t_{2}^{\alpha} \theta\right)-T\left(t_{1}^{\theta}\right)
$$

$$
=\int_{t_{1}}^{t_{2}} \alpha t^{\alpha-1} \theta A T\left(t^{\alpha} \theta\right) d t
$$

and by (7) and Lemma 5, we have

$$
\begin{aligned}
& \left\|\left(E_{\alpha}\left(t_{2}\right)-E_{\alpha}\left(t_{1}\right)\right) \chi\right\|_{H^{\nu}} \\
= & \left\|A_{\nu}\left(E_{\alpha}\left(t_{2}\right)-E_{\alpha}\left(t_{1}\right)\right) \chi\right\| \\
= & \left\|\int_{0}^{\infty} \zeta_{\alpha}(\theta) A_{\nu}\left(T\left(t_{2}^{\alpha} \theta\right)-T\left(t_{1}^{\theta}\right)\right) \chi d \theta\right\| \\
\leq & \int_{0}^{\infty} \alpha \theta \zeta_{\alpha}(\theta) \int_{t_{1}}^{t_{2}} t^{\alpha-1}\left\|A_{2+\nu} T\left(t^{\alpha} \theta\right) \chi\right\|_{L^{2}} d t d \theta \\
\leq & \int_{0}^{\infty} C_{\nu} \alpha \theta^{-\frac{\nu}{2}} \zeta_{\alpha}(\theta)\left(\int_{t_{1}}^{t_{2}} t^{-\frac{\alpha \nu}{2}}-1 d t\right)\|\chi\| d \theta \\
= & \frac{2 C_{\nu} \Gamma\left(1-\frac{\nu}{2}\right)}{\nu \Gamma\left(1-\frac{\alpha \nu}{2}\right)}\left(t_{1}^{-\frac{\alpha \nu}{2}}-t_{2}^{-\frac{\alpha \nu}{2}}\right)\|\chi\| \\
\leq & \frac{2 C_{\nu} \Gamma\left(1-\frac{\nu}{2}\right)}{\nu T_{0}^{\alpha \nu} \Gamma\left(1-\frac{\alpha \nu}{2}\right)}\left(t_{2}-t_{1}\right)^{\frac{\alpha \nu}{2}}\|\chi\|, \chi \in L^{2}(D) .
\end{aligned}
$$

Also

$$
\begin{aligned}
& \left\|\left(E_{\alpha, \alpha}\left(t_{2}\right)-E_{\alpha, \alpha}\left(t_{1}\right)\right) \chi\right\|_{H^{\nu}} \\
& =\left\|A_{\nu}\left(E_{\alpha, \alpha}\left(t_{2}\right)-E_{\alpha, \alpha}\left(t_{1}\right)\right) \chi\right\| \\
& =\left\|\int_{0}^{\infty} \alpha \theta \zeta_{\alpha}(\theta) A_{\nu}\left(T\left(t_{2}^{\alpha} \theta\right)-T\left(t_{1}^{\theta}\right)\right) \chi d \theta\right\| \\
& \leq \int_{0}^{\infty} \alpha^{2} \theta^{2} \zeta_{\alpha}(\theta) \int_{t_{1}}^{t_{2}} t^{\alpha-1}\left\|A_{2+\nu} T\left(t^{\alpha} \theta\right) \chi\right\|_{L^{2}} d t d \theta \\
& \leq \int_{0}^{\infty} C_{\nu} \alpha^{2} \theta^{1-\frac{\nu}{2}} \zeta_{\alpha}(\theta)\left(\int_{t_{1}}^{t_{2}} t^{-\frac{\alpha \nu}{2}-1} d t\right)\|\chi\| d \theta \\
& =\frac{2 \alpha C_{\nu} \Gamma\left(2-\frac{\nu}{2}\right)}{\nu \Gamma\left(1+\alpha\left(1-\frac{\nu}{2}\right)\right)}\left(t_{1}^{-\frac{\alpha \nu}{2}}-t_{2}^{-\frac{\alpha \nu}{2}}\right)\|\chi\| \\
& \leq \frac{2 C_{\nu} \Gamma\left(2-\frac{\nu}{2}\right)}{\nu T_{0}^{\alpha \nu} \Gamma\left(1+\alpha\left(1-\frac{\nu}{2}\right)\right)}\left(t_{2}-t_{1}\right)^{\frac{\alpha \nu}{2}}\|\chi\|, \chi \in L^{2}(D) .
\end{aligned}
$$

It is obviously to see that the term

$$
\left\|\left(E_{\alpha}\left(t_{2}\right)-E_{\alpha}\left(t_{1}\right)\right) \chi\right\|_{H^{\nu}} \rightarrow 0,
$$

and

$$
\left\|\left(E_{\alpha, \alpha}\left(t_{2}\right)-E_{\alpha, \alpha}\left(t_{1}\right)\right) \chi\right\|_{H^{\nu}} \rightarrow 0,
$$

as $t_{1} \rightarrow t_{2}$ which mean that the operators $E_{\alpha}(t)$ and $E_{\alpha, \alpha}(t)$ are strongly continuous.

\section{Existence results}

In this section, we present our main results on the existence of mild solutions of problem (5) and we define the following space

$$
K=\left\{u: u \in C\left([0, T], H^{\nu}\right), \sup _{t \in[0, T]}\|u\|<\infty\right\} .
$$

To do this, we make the following hypotheses:

$\left(H_{1}\right) A$ is the infinitesimal generator of a strongly continuous semigroup $\{T(t), t \geq 0\}$ on $H$. We will also suppose that the operator $E_{\alpha}(t), t>0$ is compact.

$\left(H_{2}\right)$ The function $g: \Omega \times H \rightarrow L_{0}^{2}$ satisfies the following global Lipshitz and growth conditions:

$\|g(v)\|_{L_{0}^{2}} \leq C\|u\|,\|g(u)-g(v)\|_{L_{0}^{2}} \leq C\|u-v\|$,

for any $u \in H, v \in H$. 
$\left(H_{3}\right)$ The initial value $u_{0}: \Omega \rightarrow H^{\nu}$ is a $F_{0}$-measurable random variable, it hold that

$$
\left\|u_{0}\right\|_{L^{p}\left(\Omega, H^{\nu}\right)}<\infty, \text { for any } 0 \leq \nu<\alpha<2 .
$$

$\left(H_{4}\right)$ The function $h: L_{0}^{2} \rightarrow L_{0}^{2}$ is continuous and there exists $L_{h}>0$ such that

$$
\begin{gathered}
E\left\|h\left(u_{1}(t)\right)-h\left(u_{2}(t)\right)\right\|_{L_{0}^{2}}^{p} \leq L_{h}\left\|u_{1}(t)-u_{2}(t)\right\|_{L_{0}^{2}}^{p}, \\
t \in[0, T], u_{1}, u_{2} \in L_{0}^{2},
\end{gathered}
$$

and

$$
E\|h(u(t))\|_{L_{0}^{2}}^{p} \leq L_{h} E\|u(t)\|_{L_{0}^{2}}^{p}, t \in[0, T], u \in L_{0}^{2} .
$$

$\left(H_{5}\right)$ Let $C>0$ be a real number, then the bounded bilinear operator $B: L^{2}(D) \rightarrow H^{-1}(D)$ satisfies the following properties

$$
\|B(u)\|_{H^{-1}} \leq C\|u\|^{2},
$$

and

$$
\|B(u)-B(v)\|_{H^{-1}} \leq C(\|u\|+\|v\|)\|u-v\|,
$$

for any $u, v \in L^{2}(D)$.

Definition 7. (see [2]) Let $C$ be a bounded set in metric space $(X, d)$, then Kuratowskii measure of noncompactness, $\alpha(C)$ is defined as

$\inf \{\epsilon: C$ covered by finitely many sets

such that the diameter of each set\}.

Definition 8. (see [2]). Let $\Phi: D(\Phi) \subset X \rightarrow X$ a bounded and continuous operator on Banach space $X$. Then $\Phi$ is called a condensing map if $\alpha(\Phi(Y))<$ $\alpha(Y)$ all bounded sets $Y$, where $\alpha$ denotes the Kuratowski measure of noncompactness.

Lemma 9 ([24], Example 11.7). The map $G_{1}+G_{2}$ is a $k$-set contraction with $0 \leq k<1$ and is thus condensing, if

(i) $G_{1}, G_{2}: D \subseteq X \rightarrow X$ are operators on the Banach space,

(ii) $G_{1}$ is $k$-contractive, that is

$$
\left\|G_{1} u-G_{1} v\right\| \leq k\|u-v\|,
$$

for all $u, v \in D$ and fixed point $k \in[0,1)$,

(iii) $G_{2}$ is compact.
Our main results is based on the following Sadovskii fixed points theorem.

Theorem 10. [20](Sadovskii's fixed point theorem) Let $Y$ be a convex, bounded, and closed subset of a Banach space $X$ and let $\Phi: Y \rightarrow Y$ a condensing map. Then $\Phi$ has a fixed point.

In the proof of main result, we need the following Lemmas.

Lemma 11. Assume that conditions $\left(H_{1}\right)$ and $\left(H_{2}\right)$ hold. Let $\Phi_{1}$ be the operator defined by for each $u \in$ K

$$
\Phi_{1}(u)=\int_{0}^{t}(t-s)^{\alpha-1} E_{\alpha, \alpha}(t-s) B(u(s)) d s .
$$

Then $\Phi_{1}$ is continuous and maps $K$ into itself.

Proof. It is obvious that $\Phi_{1}$ is continuous. Next we show that $\Phi_{1}(K) \subset K$. By $\left(H_{1}\right)$ and $\left(H_{2}\right)$, from the equation (11) and by applying Holder inequality, we have

$$
\begin{aligned}
& E\left\|\left(\Phi_{1} u\right)(t)\right\|_{H^{\nu}}^{p} \\
& =E\left\|\int_{0}^{t}(t-s)^{\alpha-1} A_{1} E_{\alpha, \alpha}(t-s) A_{\nu-1} B(u(s)) d s\right\|_{H^{\nu}}^{p} \\
& \leq C_{\alpha}^{p}\left(\int_{0}^{t}(t-s)^{\frac{p\left(\frac{\alpha-1}{2}\right)}{p-1}} d s\right)^{p-1} \int_{0}^{t} E\left[\left\|A_{\nu-1} B(u(s))\right\|^{p}\right] d s \\
& \leq C^{p} C_{\alpha}\left[\frac{2(p-1)}{p-2}\right]^{p-1}(T)^{\frac{p-2}{2}} \int_{0}^{t} E\left[\|u(t)\|_{H^{\nu}}^{p}\right]
\end{aligned}
$$

$=\gamma_{1} \int_{0}^{t} E\left[\|u(s)\|_{H^{\nu}}^{p}\right] d s$

where $\gamma_{1}=C^{p} C_{\alpha}\left[\frac{2(p-1)}{p-2}\right]^{p-1}(T)^{\frac{p-2}{2}}$. This complete the proof.

Lemma 12. Assume that conditions $\left(H_{1}\right)$ and $\left(H_{2}\right)$ hold. Let $\Phi_{2}$ be the operator defined by for each $u \in$ K

$$
\Phi_{2}(u)=\int_{0}^{t} S_{\alpha}(t-s) f(u(s)) d W(s) .
$$

Then $\Phi_{2}$ is continuous and maps $K$ into itself. 
Proof. Making use of the Holder inequality and Lemma 2, we obtain

$$
\begin{aligned}
& E\left\|\left(\Phi_{2} u\right)(t)\right\|_{H^{\nu}}^{p} \\
& =E\left\|\int_{0}^{t}(t-s)^{\alpha-1} E_{\alpha, \alpha}(t-s) g(u(s)) d W(s)\right\|_{H^{\nu}}^{p} \\
& \leq C(p) E\left[\left(\int_{0}^{t}\left\|(t-s)^{\alpha-1} E_{\alpha, \alpha}(t-s)\right\|^{2}\left\|A_{\nu} g(u)\right\|_{L_{0}^{2}}^{2} d s\right)\right. \\
& \leq C(p) C_{\alpha}^{p}\left(\int_{0}^{t}(t-s)^{\frac{2 p(\alpha-1)}{p-2}}\right)^{\frac{p-2}{2}} \int_{0}^{t} E\left\|A_{\nu} g(u)\right\|_{L_{0}^{2}}^{p} d s \\
& \leq C(p) C_{\alpha}^{p}\left(\frac{p-2}{p(2 \alpha-1)-2}\right)^{\frac{p-2}{2}} \int_{0}^{t} E\left\|A_{\nu} g(u)\right\|_{L_{0}^{2}}^{p} d s \\
& =\gamma_{2} \int_{0}^{t} E\left[\|u(s)\|_{H^{\nu}}^{p}\right] d s,
\end{aligned}
$$

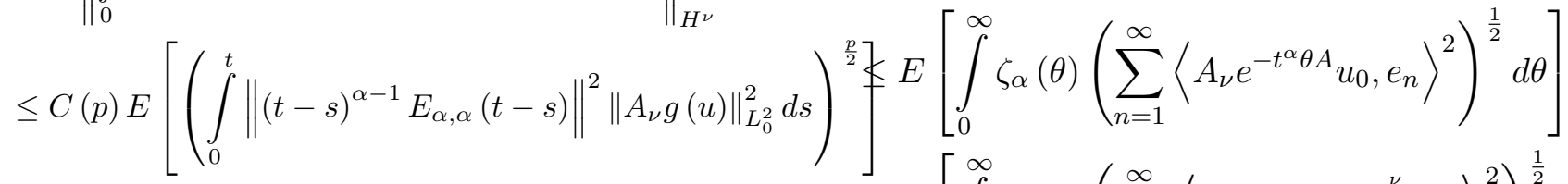

where $\gamma_{2}=C(p) C_{\alpha}^{p} C^{p}\left[\frac{p-2}{p(2 \alpha-1)-2}\right]^{\frac{p-2}{2}}$.

That is $\Phi_{2}(B) \subset B$.

Lemma 13. Assume that conditions $\left(H_{1}\right)$ and $\left(H_{4}\right)$ hold. Let $\Phi_{3}$ be the operator defined by for each $u \in$ $B$

$$
\left(\Phi_{3} u\right)(t)=E_{\alpha}(t) u_{0}+h(u(t))
$$

Then $\Phi_{3}$ is continuous and maps $K$ into $K$.

Proof. The continuity in $p-t h$ moment of $\Phi_{3}$ follows from $\left(H_{4}\right)$.

Next, we show that $\Phi_{3}(Y) \subset Y$. By $\left(H_{1}\right),\left(H_{5}\right)$ and from (13), we have

$$
E\left\|\left(\Phi_{3} u\right)(t)\right\|_{L_{0}^{2}}^{p} \leq E\|h(u(t))\|_{L_{0}^{2}}^{p} \leq L_{h} E\|u(t)\|_{L_{0}^{2}}^{p} .
$$

So, we conclude $\Phi_{3}(K) \subset K$.

Lemma 14. Assume that conditions $\left(H_{1}\right)$ and $\left(H_{2}\right)$ hold. Then

$$
E\left[\left\|E_{\alpha}(t) u_{0}\right\|_{H^{\nu}}\right] \leq E\left[\left\|u_{0}\right\|_{H^{\nu}}\right]
$$

Proof. By Lemma 5, we have

$$
\begin{aligned}
& E\left[\left\|E_{\alpha}(t) u_{0}\right\|_{H^{\nu}}\right] \\
& \leq E\left[\int_{0}^{\infty} \zeta_{\alpha}(\theta)\left(\left\|A_{\nu} T\left(t^{\alpha} \theta\right) u_{0}\right\|^{2}\right)^{\frac{1}{2}} d \theta\right] \\
& \leq E\left[\int_{0}^{\infty} \zeta_{\alpha}(\theta)\left(\sum_{n=1}^{\infty}\left\langle A_{\nu} u_{0}, e^{-t^{\alpha} \theta \lambda_{n}^{\frac{\nu}{2}}}, e_{n}\right\rangle^{2}\right)^{\frac{1}{2}} d \theta\right] \\
& \leq E\left[\int_{0}^{\infty} \zeta_{\alpha}(\theta)\left\|u_{0}\right\|_{H^{\nu}} d \theta\right]=E\left[\left\|u_{0}\right\|_{H^{\nu}}\right] \text {. }
\end{aligned}
$$

First, we define a map $F: K \rightarrow C\left([0, T], H^{\nu}\right)$ in the following manner: for any $u \in K$,

$$
\begin{aligned}
(F u)(t)= & \int_{0}^{t}(t-s)^{\alpha-1} E_{\alpha, \alpha}(t-s) B(u(s)) d s \\
& +\int_{0}^{t}(t-s)^{\alpha-1} E_{\alpha, \alpha}(t-s) f(u(s)) d W(s) \\
& +E_{\alpha}(t) u_{0}(s)+h(u(t)) .
\end{aligned}
$$

Now, we set $F=F_{1}+F_{2}$, where

$$
\left(F_{1} u\right)(t)=E_{\alpha}(t) u_{0}(s)+h(u(t)),
$$

and

$$
\begin{aligned}
\left(F_{2} u\right)(t)= & \int_{0}^{t}(t-s)^{\alpha-1} E_{\alpha, \alpha}(t-s) B(u(s)) d s \\
& +\int_{0}^{t}(t-s)^{\alpha-1} E_{\alpha, \alpha}(t-s) f(u(s)) d W(s),
\end{aligned}
$$

for $t \in[0, T]$.

Lemma 15. Assume $\left(H_{2}\right),\left(H_{4}\right),\left(H_{5}\right)$ hold and $0<$ $\nu<\alpha \leq 2, p \geq 2$, Then

$E\left\|E_{\alpha}\left(t_{2}\right)-E_{\alpha}\left(t_{1}\right)\right\|_{H^{\nu}}^{p} \leq C_{\alpha, \nu}^{p}\left(t_{2}-t_{1}\right)^{\frac{\alpha \nu}{2}} E\left\|u_{0}\right\|^{p}$.

Proof. We set

$$
I_{1}=F_{1}\left(t_{2}\right)-F_{1}\left(t_{1}\right)=E_{\alpha}\left(t_{2}\right) u_{0}-E_{\alpha}\left(t_{2}\right) u_{0}
$$


For any $p \geq 2$, by vertue of Lemma 6 , it follows that

$$
\begin{aligned}
& E\left[\left\|I_{1}\right\|_{H^{\nu}}^{p}\right]=E\left[A\left\|E_{\alpha}\left(t_{2}\right) u_{0}-E_{\alpha}\left(t_{2}\right) u_{0}\right\|^{p}\right] \\
& \leq C_{\alpha, \nu}^{p}\left(t_{2}-t_{1}\right)^{\frac{\alpha \nu}{2}} E\left\|u_{0}\right\|^{p} .
\end{aligned}
$$

It is obviously to see that the term $\left\|\left(F_{1}\left(t_{2}\right)-F_{1}\left(t_{1}\right)\right)\right\|_{Y} \rightarrow 0$ as $t_{1} \rightarrow t_{2}$ which mean that the operators $F_{1}$ is strongly continuous.

Lemma 16. Assume $\left(H_{2}\right),\left(H_{4}\right),\left(H_{5}\right)$ hold and $0<$ $\nu<\alpha \leq 2, p \geq 2$, then the operator $F_{2}$ is uniformly bounded.

Proof. From Lemma 11, Lemma 12 and by means of extension of Gronwall's lemma, we have

$$
\sup _{t \in[0, T]} E\left[\left\|F_{2}(u(t))\right\|_{H^{\nu}}^{p}\right] \leq \infty,
$$

that is the operator $F_{2}$ is uniformly bounded.

Lemma 17. Assume $\left(H_{2}\right),\left(H_{4}\right),\left(H_{5}\right)$ hold and $0<$ $\nu<\alpha \leq 2, p \geq 2$. Then the operator $F_{2}$ is equicontinuous.

Proof. For any $0 \leq t_{1}<t_{2} \leq T$, from

$$
\begin{aligned}
& \left(F_{2} u\right)\left(t_{2}\right)-\left(F_{2} u\right)\left(t_{1}\right) \\
& =\int_{0}^{t_{2}}\left(t_{2}-s\right)^{\alpha-1} E_{\alpha, \alpha}\left(t_{2}-s\right) B(u(s)) d s \\
& -\int_{0}^{t_{1}}\left(t_{1}-s\right)^{\alpha-1} E_{\alpha, \alpha}\left(t_{1}-s\right) B(u(s)) d s \\
& +\int_{0}^{t_{2}}\left(t_{2}-s\right)^{\alpha-1} E_{\alpha, \alpha}\left(t_{2}-s\right) g(u) d W(s) \\
& -\int_{0}^{t_{1}}\left(t_{1}-s\right)^{\alpha-1} E_{\alpha, \alpha}\left(t_{1}-s\right) g(u) d W(s)=I_{2}+I_{3},
\end{aligned}
$$

where

$$
\begin{aligned}
I_{2} & =\int_{0}^{t_{2}}\left(t_{2}-s\right)^{\alpha-1} E_{\alpha, \alpha}\left(t_{2}-s\right) B(u(s)) d s \\
& -\int_{0}^{t_{1}}\left(t_{1}-s\right)^{\alpha-1} E_{\alpha, \alpha}\left(t_{1}-s\right) B(u) d(s) \\
& \int_{0}^{t_{1}}\left(t_{1}-s\right)^{\alpha-1}\left[E_{\alpha, \alpha}\left(t_{2}-s\right)\right. \\
& +\int_{0}^{t_{1}}\left[\left(t_{2}-s\right)^{\alpha-1}-\left(t_{1}-s\right)^{\alpha-1}\right] \\
& \times E_{\alpha, \alpha}\left(t_{2}-s\right) B(u(s)) d s \\
& +\int_{t_{1}}^{t_{2}}\left(t_{2}-s\right)^{\alpha-1} E_{\alpha, \alpha}\left(t_{2}-s\right) B(u(s)) d s \\
& =I_{21}+I_{22}+I_{23},
\end{aligned}
$$

and

$$
\begin{aligned}
I_{3}= & \int_{0}^{t_{2}}\left(t_{2}-s\right)^{\alpha-1} E_{\alpha, \alpha}\left(t_{2}-s\right) f(u(s)) d W s \\
& -\int_{0}^{t_{1}}\left(t_{1}-s\right)^{\alpha-1} E_{\alpha, \alpha}\left(t_{1}-s\right) f(u) d W(s) \\
= & \int_{0}^{t_{1}}\left(t_{1}-s\right)^{\alpha-1}\left[E_{\alpha, \alpha}\left(t_{2}-s\right)-E_{\alpha, \alpha}\left(t_{1}-s\right)\right] f(u(s)) d W(s) \\
& +\int_{0}^{t_{1}}\left[\left(t_{2}-s\right)^{\alpha-1}-\left(t_{1}-s\right)^{\alpha-1}\right] E_{\alpha, \alpha}\left(t_{2}-s\right) f(u(s)) d W(s) \\
& +\int_{t_{1}}^{t_{2}}\left(t_{2}-s\right)^{\alpha-1} E_{\alpha, \alpha}\left(t_{2}-s\right) f(u(s)) d W(s) \\
= & I_{31}+I_{32}+I_{33} .
\end{aligned}
$$

For the first term $I_{21}$ in (15), applying the assumptions $\left(H_{5}\right)$ and Lemma 10 and Holder inequality, we 
have

$$
\begin{aligned}
& E=\left[\left\|I_{21}\right\|_{H^{v}}^{p}\right] \\
& =E\left[\| \int_{0}^{t_{1}}\left(t_{1}-s\right)^{\alpha-1}\left[E_{\alpha, \alpha}\left(t_{2}-s\right)\right.\right. \\
& \left.\left.-E_{\alpha, \alpha}\left(t_{1}-s\right)\right] B(u(s)) d s \|^{P}\right] \\
& \leq C_{\alpha \nu}^{p}\left(t_{2}-t_{1}\right)^{\frac{p \alpha(\nu+1)}{2}} \times \\
& \left(\int_{0}^{t_{1}}\left(t_{1}-s\right)^{\frac{p(\alpha-1)}{p-1}} d s\right)^{p-1} \int_{0}^{t} E\left[\left\|A_{-1} B(u(s))\right\|_{H^{1}}^{p}\right] d s \\
& \leq C^{p} C_{\alpha \nu}^{p} T^{p \alpha} \times \\
& \left(\frac{p-1}{p \alpha-1}\right)^{p-1}\left(\sup _{t \in[0, T]} E\left[\|u(s)\|_{H^{1}}^{2 p}\right]\right)\left(t_{2}-t_{1}\right)^{\frac{p \alpha(\nu+1)}{2}} \text {. }
\end{aligned}
$$

Using the assumptions $\left(H_{5}\right)$ and Lemma 10 and Holder inequality, we have

$$
\begin{aligned}
& E\left[\left\|I_{22}\right\|_{H^{\nu}}^{p}\right] \\
& =E\left[\| \int_{0}^{t_{1}}\left[\left(t_{2}-s\right)^{\alpha-1}-\left(t_{1}-s\right)^{\alpha-1}\right]\right. \\
& \left.\quad \times\left[A_{v} E_{\alpha, \alpha}\left(t_{2}-s\right)\right] B(u(s)) d s \|^{p}\right] \\
& \leq C_{\alpha}^{p}\left(\int _ { 0 } ^ { t _ { 1 } } \left\{\left[\left(t_{2}-s\right)^{\alpha-1}-\left(t_{1}-s\right)^{\alpha-1}\right]\right.\right. \\
& \left.\left.\times\left(t_{2}-s\right)^{\frac{-\alpha(\nu+1)}{2}}\right\} d s\right)^{p-1} \int_{0}^{t} E\left[\left\|A_{-1} B(u(s))\right\|_{H^{1}}^{p}\right] d s \\
& \quad \leq C^{p} C_{\alpha}^{p} T\left(\frac{p-1}{p\left(\alpha-\frac{\alpha(\nu+1)}{2}\right)}\right)^{p-1} \\
& \times\left(\sup _{t \in[0, T]} E\left[\|u(s)\|_{H^{1}}^{2 p}\right]\right)\left(t_{2}-t_{1}\right)^{\frac{p \alpha(1-\nu)-2}{2}},
\end{aligned}
$$

and

$$
\begin{aligned}
& E\left[\left\|I_{23}\right\|_{H^{\nu}}^{p}\right] \\
& =E\left[\left\|\int_{t_{1}}^{t_{2}}\left(t_{2}-s\right)^{\alpha-1} A_{\nu} E_{\alpha, \alpha}\left(t_{2}-s\right) B(u(s)) d s\right\|^{p_{1}}\right] \\
& \leq C_{\alpha}^{p}\left(\int_{t_{1}}^{t_{2}}\left(t_{2}-s\right)^{\alpha-1-\frac{\alpha(\nu+1)}{2}} d s\right)^{p-1} \\
& \quad \times \int_{t_{1}}^{t_{2}\left[\left\|A_{v} B(u(s))\right\|_{H^{1}}^{p}\right] d s} \\
& \leq C^{p} C_{\alpha}^{p}\left(\frac{p-1}{p\left(\alpha-\frac{\alpha(\nu+1)}{2}\right)-1}\right)^{p-1} \\
& \quad \times\left(\begin{array}{c}
s u p E\left[\|u(s)\|_{H^{1}}^{2 p}\right] \\
t \in[0, T]
\end{array}\right)\left(t_{2}-t_{1}\right)^{\frac{p \alpha(1-\nu)}{2}} .
\end{aligned}
$$

Next, by following similar arguments as in the proof of (17)-(19) and using Lemma 2 there holds,

$$
\begin{aligned}
& E\left[\left\|I_{31}\right\|_{H^{\nu}}^{p}\right] \\
& =E\left[\| \int_{0}^{t_{1}}\left(t_{1}-s\right)^{\alpha-1}\left[E_{\alpha, \alpha}\left(t_{2}-s\right)\right.\right. \\
& \left.\left.-E_{\alpha, \alpha}\left(t_{1}-s\right)\right] f(u(s)) d W s \|^{P}\right] \\
& \leq C(p) E\left[\left(\int_{0}^{t_{1}} \|\left(t_{1}-s\right)^{\alpha-1} A_{v}\left[E_{\alpha, \alpha}\left(t_{2}-s\right)\right.\right.\right. \\
& \left.\left.\left.-E_{\alpha, \alpha}\left(t_{1}-s\right)\right]\left\|^{2}\right\| f(u(s)) \|_{L_{0}^{2}}^{2} d s\right)^{\frac{p}{2}}\right] \\
& \leq C(p) C_{\alpha \nu}^{p}\left(t_{2}-t_{1}\right)^{\frac{p \alpha \nu}{2}}\left(\int_{0}^{t_{1}}\left(t_{1}-s\right)^{\frac{2 p(\alpha-1)}{p-2}} d s\right)^{\frac{p-2}{2}} \\
& \times \int_{0}^{t_{1}} E\|f(u(s))\|_{L_{0}^{2}}^{p} d s \\
& \leq C^{p} C_{\alpha \nu}^{p} T^{\frac{2 p \alpha-p-1}{2}}\left(\frac{p-1}{2 p \alpha-p-2}\right)^{p-1} \\
& \times\left(\sup _{t \in[0, T]} E\left[\|u(s)\|^{p}\right]\right)\left(t_{2}-t_{1}\right)^{\frac{p \alpha \nu}{2}},
\end{aligned}
$$


and

$$
\begin{aligned}
& E\left[\left\|I_{32}\right\|_{H^{\nu}}^{p}\right] \\
& =E\left[\| \int_{0}^{t_{1}}\left[\left(t_{2}-s\right)^{\alpha-1}-\left(t_{1}-s\right)^{\alpha-1}\right]\right. \\
& \left.\times\left[A_{v} E_{\alpha, \alpha}\left(t_{2}-s\right)\right] f(u(s)) q W s \|_{L_{0}^{2}}^{p}\right] \\
& \leq C(p) \times \\
& E\left[\left(\int_{0}^{t_{1}}\left\|\left[\left(t_{2}-s\right)^{\alpha-1}-\left(t_{1}-s\right)^{\alpha-1}\right]\left[A_{v} E_{\alpha, \alpha}\left(t_{2}-s\right)\right]\right\|\right.\right. \\
& \left.\left.\times\|f(u(s))\|_{L_{0}^{2}}^{2} d s\right)^{\frac{p}{2}}\right] \\
& \leq C(p) C_{\alpha}^{p} \times \\
& \left(\int_{0}^{t_{1}}\left\{\left[\left(t_{2}-s\right)^{\alpha-1}-\left(t_{1}-s\right)^{\alpha-1}\right]\left(t_{2}-s\right)^{\frac{-\alpha \nu}{2}}\right\}^{\frac{2 p}{p-2}} d s\right) \\
& \times \int_{0}^{t} E\left[\|f(u(s))\|_{L_{0}^{2}}^{p}\right] d s \\
& \leq C(p) C^{p} C_{\alpha}^{p} T\left(\frac{2(p-2)}{2 p \alpha(2-\nu)-2(p+2)}\right)^{\frac{p-2}{2}} \\
& \times\left(\sup _{t \in[0, T]} E\left[\|u(t)\|^{p}\right]\right)\left(t_{2}-t_{1}\right)^{\frac{2 p \alpha(2-\nu)-2(p+2)}{4}},
\end{aligned}
$$

and

$$
\begin{aligned}
& E\left[\left\|I_{33}\right\|_{H^{\nu}}^{p}\right] \\
& \left.=E\left[\left\|\int_{t_{1}}^{t_{2}}\left(t_{2}-s\right)^{\alpha-1} A_{\nu} E_{\alpha, \alpha}\left(t_{2}-s\right) B(u(s)) d s\right\|^{p}\right]\right] \begin{array}{l}
\text { so } \\
\text { the } \\
\text { we }
\end{array} \\
& \leq C(p) \times \\
& E\left[\left(\int_{0}^{t_{1}}\left\|\left[\left(t_{2}-s\right)^{\alpha-1}-\left(t_{1}-s\right)^{\alpha-1}\right]\left[A_{v} E_{\alpha, \alpha}\left(t_{2}-s\right)\right]\right\|^{2}\right.\right. \\
& \left.\left.\quad \times\|f(u(s))\|_{L_{0}^{2}}^{2} d s\right)^{\frac{p}{2}}\right] \\
& \quad \times \int_{t_{1}}^{t_{2}} E\left[\|f(u(s))\|_{L_{0}^{2}}^{p}\right] d s \\
& \leq C(p) C^{p} C_{\alpha}^{p}\left(\frac{2(p-2)}{2 p \alpha(2-\nu)-2(p+2)}\right)^{\frac{p-2}{2}} \\
& \times\left(\sup _{t \in[0, T]}^{t_{2}} E\left[\|u(t)\|^{p}\right]\right)\left(t_{2}-t_{1}\right)^{\frac{2 p \alpha(2-\nu)-2 p}{4}}
\end{aligned}
$$

Taking expectation on the both side of (14) and in view of estimates (15) and (17)-(22), we conclude that

$$
\left\|\left(F_{2} u\right)\left(t_{2}\right)-\left(F_{2} u\right)\left(t_{1}\right)\right\|_{L^{p}\left(\Omega, H^{\nu}\right)} \leq C\left(t_{2}-t_{1}\right)^{\gamma},
$$

where $\gamma=\min \left\{\frac{\alpha \nu}{2}, \frac{\alpha p(1-\nu)-2}{2 p}, \frac{2 p \alpha(2-\nu)-2(p+2)}{4 p}\right\}$ when $0<t_{2}-t_{1}<1$.

Otherwise, if $t_{2}-t_{1} \geq 1$, then we set $\gamma=$ $\max \left\{\frac{\alpha(\nu+1)}{2}, \frac{\alpha(2-\nu-1)}{2}, \frac{2 p \alpha(2-\nu)-2 p}{4 p}\right\}$.

Lemma 18. Assume the conditions $\left(H_{1}\right)$ and $\left(H_{2}\right)$ hold. Then $F$ maps $K$ into itself.

Proof. Let the nonlinear operator $F$ defined by, for $\underline{t}_{2} \geq 0$,

$$
\begin{aligned}
(F u)(t)= & E_{\alpha}(t) u_{0}+h(u(t)) \\
& +\int_{0}^{t}(t-s)^{\alpha-1} E_{\alpha, \alpha}(t-s) B(u(s)) d s \\
& +\int_{0}^{t}(t-s)^{\alpha-1} E_{\alpha, \alpha}(t-s) g(u) d W(s) .
\end{aligned}
$$

We prove that the operator $F$ has a fixed point, which is a mild solution of the problem (1)-(3). We shall employ Theorem 10. For better readability, we break the proof into a sequence of steps.

Step 1. For $0<\lambda<1$, set $\{u \in K: u=\lambda F u\}$ is bounded.

Let $u \in K$ be a possible solution of $u=\lambda F u$ for some $0<\lambda<1$. Then by $\left(H_{1}\right)-\left(H_{4}\right)$ and applying the similar arguments in Lemma 13 and Lemma 16 , we have

$$
\begin{aligned}
E\|u(t)\|_{H^{\nu}}^{p} \leq & 3^{p-1}\left\|E_{\alpha}(t) u_{0}\right\|_{H^{\nu}}^{p} \\
& +3^{p-1}\|h(u(t))\|_{H^{\nu}}^{p} \\
& +3^{p-1} E\left\|\Phi_{1}(u(t))\right\|_{H^{\nu}}^{p} \\
& +3^{p-1} E\left\|\Phi_{2}(u(t))\right\|_{H^{\nu}}^{p} \\
\leq & 3^{p-1} E\left[\left\|u_{0}\right\|_{H^{\nu}}\right] \\
& +3^{p-1}\left(\gamma_{1}+\gamma_{2}\right) \int_{0}^{t} E\left[\|u\|_{H^{\nu}}^{p}\right] d s .
\end{aligned}
$$

By means of the extention of Gronwall's lemma, it holds that

$$
\sup _{t \in[0, T]} E\|u(t)\|_{H^{\nu}}^{p}<\infty .
$$

This indicates that $u(\cdot)$ is bounded on $[0, T]$.

Step 2. $F: K \rightarrow C\left([0, T], H^{\sigma}\right)$ is continuous. 
Let $\left\{u_{n}(t)\right\}_{n \geq 0}$ with $u_{n} \rightarrow u(n \rightarrow \infty)$ in $K$. Then there is a number $r>0$ such that $E\left\|u_{n}(t)\right\|_{H^{\nu}}^{2} \leq r$ for all $n$ and a.e. $t \in[0, T]$, so $u_{n} \in B_{r}(0, Y)=$ $\left\{u \in K: \sup _{t \in[0, T]}\|u\|_{H^{\sigma}}\right\}$ and $u \in B_{r}(0, K)$. By the assumptions $\left(\mathrm{H}_{2}\right)$ and similar argument to obtain (12) and (13), we have

$$
\begin{aligned}
E & \left\|\left(F u_{n}\right)(t)-(F u)(t)\right\|_{H^{\nu}}^{p} \\
\leq & 3^{p-1}\left\|h\left(u_{n}(t)\right)-h(u(t))\right\|_{H^{\nu}}^{P} \\
& +3^{p-1} E\left\|\Phi_{1}\left(u_{n}(t)-u(t)\right)\right\|_{H^{\nu}}^{p} \\
& +3^{p-1} E\left\|\Phi_{2}\left(u_{n}(t)-u(t)\right)\right\|_{H^{\nu}}^{p} \\
\leq & 3^{p-1}\left\|h\left(u_{n}(t)\right)-h(u(t))\right\|_{H^{\nu}}^{p} \\
& +3^{p-1}\left(G \gamma_{1}+K \gamma_{2}\right)\left(\int_{0}^{t} E\left\|u_{n}-u\right\|_{H^{\nu}}^{p} d s\right) .
\end{aligned}
$$

Then, we have for all $t \in[0, T]$,

$$
\left\|F u_{n}-F u\right\|_{Y}^{p} \longrightarrow 0 \text {, as } n \longrightarrow \infty .
$$

Therefore $F$ is continuous.

Step 3. We decompose $F$ as $F=F_{1}+F_{2}$ where $F_{1}$ and $F_{2}$ defined above.

(1) $F_{1}$ is a contraction on $K$. Let $u, v \in K$. It follows from Lemma 2 that

$$
\begin{gathered}
E\left\|F_{1} u-F_{1} v\right\|_{H^{\nu}}^{p} \leq L_{h} E\|u(s)-v(s)\|_{H^{\nu}}^{p} \\
\leq L_{h} \sup _{s \in[0, T]} E\|u(s)-v(s) d s\|_{H^{\nu}}^{p} \\
\leq L_{h}\|u(s)-v(s) d s\|_{Y}^{p}
\end{gathered}
$$

Taking supremum over $t$

$$
\left\|F_{1} u-F_{1} v\right\|_{Y}^{p} \leq L_{0}\|u(s)-v(s)\|_{Y}^{p},
$$

where $L_{0}=L_{h}<1$.

Hence $F_{1}$ is a contraction on $K$.

(2) $F_{2}$ is compact operator. Let $u, v \in K$. It follows from $\left(H_{2}\right),\left(H_{5}\right)$ and Lemma 2 that

$$
\begin{aligned}
& E\left\|F_{2} u-F_{2} v\right\|_{H^{\nu}}^{2} \\
& \leq 2^{p-1} E \| \int_{0}^{t}(t-s)^{\alpha-1} E_{\alpha, \alpha}(t-s) A_{\nu} \\
& {[g(u(s))-g(v(s))] d W(s) \|_{H^{\nu}}^{2}} \\
& \quad+2^{p-1} E \| \int_{0}^{t}(t-s)^{\alpha-1} E_{\alpha, \alpha}(t-s) A_{\nu} \\
& \quad \times[B(u(s))-B(v(s))] d s \|_{H^{\nu}}^{p} \\
& \leq\left(\gamma_{1}+\gamma_{2}\right) E\left(\int_{0}^{t}\|u-v\|_{H^{\nu}}^{2} d s\right),
\end{aligned}
$$

which implies

$$
\sup _{t \in[0, T]} E\left\|F_{2} u-F_{2} v\right\|_{H^{\nu}}^{2}=\left(\gamma_{1}+\gamma_{2}\right) \sup _{t \in[0, T]} E\|u-v\|_{H^{\nu}}^{2} .
$$

Since $0<L=\gamma_{1}+\gamma_{2}<1$, then $F$ is contraction maping on $K$.

From Lemma 16 and Lemma 17, the operator $F_{2}$ is relatively compact. together with Ascoli's theorem, we conclude that the operator $F_{2}$ is compact.

In view of Theorem 10, we conclude that $F$ has at least one fixed point, which is a mild solution of the problem (1)-(2).

\section{Conclusion}

In this paper, we study a class of a stochastic partial differential equation with noise. By using stochastic analysis theory, fractional calculations, operator semigroup theory and applying Sadovskii's fixed point theorem, we obtain the existence of mild solutions for these equation. In the future, We develop a stochastic calculus for the fractional Brownian motion with Hurst parameter in different cases using the techniques of the Malliavin calculus.

\section{References:}

[1] A. Bahar and X. Mao, Stochastic delay LotkaVolterra model. J. Math. Anal. Appl, 292, 364380 (2004)

[2] J. Banas and K. Goebel, Measure of Noncompactness in Banach Space, Lecture Notes in Pure and Applied Matyenath, Dekker, New York, 1980.

[3] T. Caraballo and K. Liu, Exponential stability of mild solutions of stochastic partial differential equations with delays, Stoch. Anal. Appl. 17 (1999), 743-763 .

[4] D. Conte, B. Paternoster, L. Moradi and F. Mohammadi, Construction of exponentially fitted explicit peer methods, International Journal of Circuits, Systems and Signal Processing, pp. 501-506, Volume 13, 2019.

[5] J. Cui and L. Yan, Existence result for fractional neutral stochastic integrodifferential equations with infinite delay, J. Phys. A 44, (2011), 335-201.

[6] T. E. Duncan, B. Maslowski and B. PasikDuncan, Semilinear stochastic equations in a Hilbert space with a fractional Brownian motion, SIAM J. Math. Anal, 40 (6) (2009), 2286-2315. 
[7] K. Ezzinbi, S. Ghnimi Local existence and global continuation for some partial functional integrodifferential equations. Afr. Diaspora $J$. Math, 12(1), (2011), 3445

[8] P. Germain, Multipliers, paramultipliers, and weak-strong uniqueness for the Navier-Stokes equations,J. Differential Equations, V.226, N.2 (2006), 373-428.

[9] Y. Jiang, T. WeiX. Zhou, Stochastic generalized Burgers equations driven by fractional noises, J. Differential Equations. 252(2)(2012), 19341961.

[10] R. Kruse, Strong and weak approximation of semilinear stochastic evolution equations, Springer, 2014.

[11] P.G. Lemarie-Rieusset, Recent developments in the NavierStokes problem, CRC Press, 2002.

[12] X. Mao, S. Sabanis and E. Renshaw, Asymptotic behaviour of the stochastic LotkaVolterra model. J. Math. Anal. Appl,

[13] X. Mao, C. Yuan and J. Zou, Stochastic differential delay equations of population dynamics. J. Math. Anal. Appl, 304, 296320 (2005) 287, 141156 (2003).

[14] X. Mao, G. Marion and E. Renshaw, Environmental Brownian noise suppresses explosions in population dynamics. Stoch. Process. Appl, 97, 95110 (2002)

[15] H. Miura, Remark on uniqueness of mild solutions to the Navier-Stokes equations, J. Funct. Anal, V.218, N.1, (2005), 110-129.

[16] Y. Ren and D.D. Sun, Second-order neutral stochastic evolution equations with infinite delay under carathodory conditions, J. Optim. Theory Appl, 147 (2010), 569-582.

[17] Y. Ren, Q. Zhou and L. Chen, Existence, uniqueness and stability of mild solutions for time-dependent stochastic evolution equations with Poisson jumps and infinite delay, J. Optim. Theory Appl, 149 (2011) 315-331.

[18] F.A. Rihan, H.J. Alsakaji, and C. Rajivganthi, Stochastic SIRC epidemic model with timedelay for COVID-19. Adv Differ Equ, 2020, 502 (2020). https://doi.org/10.1186/s13662-02002964-8

[19] Y.V. Rogovchenko, Nonlinear impulse evolution systems and applications to population models, J. Math. Anal. Appl, 207(1997), 300315.

[20] B. N. Sadovskii, On a fixed point principle, Functional Analysis and Its Applications, vol. 1, pp. 74-76, 1967.
[21] N. Samarinas, C. Tzimopoulos and C. Evangelides, Fuzzy Numerical Solution to Horizontal Infiltration, International Journal of Circuits, Systems and Signal Processing, pp. 325-332, Volume 12, 2018.

[22] T. Taniguchi, The existence of energy solutions to 2-dimensional non-Lipschitz stochastic Navier-Stokes equations in unbounded domains,J. Differential Equations, 251(12) (2011), 3329-3362.

[23] R. N. Wang, D. H. Chen and T. J. Xiao, Abstract fractional Cauchy problems with almost sectorial operators, J. Differential Equations, 252(1)(2012), 202-235.

[24] E. Zeidler, Nonlinear Functional Analysis and Its Application: Fixed Point-Theorems, vol. 1, Springer,NewYork,NY,USA, 1986.

[25] G. Zou, B. Wang, Stochastic Burgers equation with fractional derivative driven by multiplicative noise, Comput. Math. Appl. (2017) http://dx.doi.org/10.1016/ j.camwa.2017.08.023.

[26] Y. Zhou and L. Peng, On the time-fractional Navier-Stokes equations, Comput. Math. Appl, 73(6)(2017), 874-891.

[27] X. J. Yang, Advanced local fractional calculus and its applications, World Science, New York, 2012.

[28] Y. Yuan and L.J.S. Allen, Stochastic models for virus and immune system dynamics. Math. Biosci, 234, 8494 (2011)

[29] Hui Yu, Convergence of the Euler Method in Probability to SDEs under the Generalized Khasminskii-type Conditions, International Journal of Circuits, Systems and Signal Processing, pp. 251-258, Volume 13, 2019.

\section{Creative Commons Attribution License 4.0 (Attribution 4.0 International, CC BY 4.0)}

This article is published under the terms of the Creative Commons Attribution License 4.0 https://creativecommons.org/licenses/by/4.0/deed.en_US 